\title{
A PROPOSAL, FOR THE SEARCH OF EXTRASOLAR PLANETS BY OCCULTATION
}

\author{
Jean Schneider and Michel Chevreton \\ CNRS, Observatoire de Paris \\ 92195 Meudon, France
}

\begin{abstract}
Summary
We intend to search for extrasolar planets by looking for the occultations they provoke on their parent stars. Because normal (solar-type) stars give only very weak occultations with low occultation probabilities, we will investigate a class of stars more suited to this method, the dM Dwarfs. We expect show that:

1) the occultation factor is significantly enhanced

2) the probability of occultation is much large.

We therefore propose to monitor a sample of $250 \mathrm{dMs}$ during several months using a photometric telescope with a specially designed photometer.

This project has been submitted as a routine program to the solar $90 \mathrm{~cm}$ telescope THEMIS, on which we propose to permanently install the photometer. The photometer can be adapted to any photometric telescope.
\end{abstract}

Editor's Note:

The subject matter of this paper can be found in Proceedings of the 24th ESLAB Symposium on the Formation of Stars and Planets and the Evolution of the Solar System, Friedrichshafen, ed. B. Battrick, ESA SP-315, 1990, pp. 67-71. 